\title{
Percepción de la AR en una población fuera del ámbito sanitario
}

\author{
Jorge Velasco Zamora ${ }^{1,2}$, María Julia Papagno², Alejandro Benítez², Adrián Estévez ${ }^{2}$, Matías Preisegger, \\ Ricardo A Dewey ${ }^{3}$ \\ ${ }^{1}$ Fundación Articular, ${ }^{2}$ Instituto CER-Quilmes, ${ }^{3} \mathrm{IIB}-\mathrm{INTECH}$. Buenos Aires
}

\section{Resumen}

Objetivos: El acceso temprano a la población de pacientes con artritis reumatoidea (AR) a la asistencia especializada es un predictor de buen pronóstico. Sin embargo, la demora en la consulta es una constante; fueron identificados factores tales como los vinculados al paciente, el médico generalista 0 al sistema de salud. El objeto del estudio fue el de explorar la percepción de la población fuera del ámbito asistencial en un escenario potencial de sintomatología compatible con AR.

Material y métodos: Se realizó un muestreo aleatorio de 1073 individuos mayores de 18 años, se realizó en la vía pública de los partidos de Quilmes y Berazategui. La encuesta fue efectuada por voluntarias entrenadas a tal fin las que indagaron acerca de a qué profesional consultaría si tuviera dolor e inflamación de los nudillos y muñecas de ambas manos, si cree padecer estos síntomas y si conoce la diferencia entre artritis y artrosis.

Resultados: Frente a la posibilidad de padecer síntomas que remedan AR, la población encuestada eligió a los siguientes especialistas: traumatólogo $(61 \%)$, clínico $(17 \%)$, reumatólogo $(15 \%)$, otros $(7 \%)$. Un tercio de los encuestados refirió padecer esos síntomas y la mayoría $(61 \%)$ referían no conocer la diferencia entre artritis y artrosis. Quienes más eligieron al reumatólogo como opción (56\%) fueron del sexo femenino de entre 51 y 80 años y que conocían la diferencia entre AR y $0 \mathrm{~A}$.

Conclusiones: Laausencia relativa del reumatólogo en la preferencia de la población encuestada, así como la confusión respecto de la presencia de sintomatología compatible con la enfermedad 0 de la diferencia entre artritis y artrosis señalan la necesidad de ejecutar campañas de concientización pública dirigidas a la reafirmación de la especialidad frente a la patología articular inflamatoria.

Palabras clave: percepción, encuesta, reumatología, artritis reumatoidea.

\section{Abstract}

Objectives: Early access to specialized care is a predictor of good prognosis in patients recently diagnosed rheumatoid arthritis (RA). However, the delay in the consultation is a constant; they were identified factors such as those related to the patient, the general practitioner or the health system. The purpose of the study was to explore the perception of the population outside the healthcare setting in a potential scenario of symptoms compatible with RA.

Material and methods: A random sample of 1073 individuals aged 18 and over was conducted. It was held in public matches of Quilmes and Berazategui. The survey was conducted by volunteers trained for this purpose which were asked which professional (in terms of specialty) would consult if they had pain and swelling of the knuckles and wrists of both hands, and "if you know the difference between arthritis and osteoarthritis".

Results: Facing the possibility of having symptoms that mimic RA, the population surveyed chose the following specialists: orthopedic surgeons $(61 \%)$, clinicians $(17 \%)$, rheumatologists $(15 \%)$, others $(7 \%)$. A third of respondents have referred these symptoms and most $(61 \%)$ reported not knowing the difference between arthritis and osteoarthritis. People who chose rheumatologist an option (56\%) were female between 51 and 80 years and knew the difference between $\mathrm{RA}$ and $\mathrm{OA}$.

Conclusions: The relative absence of rheumatologist in the preference of the surveyed population, as well as confusion about the presence of symptoms compatible with the disease or the difference between arthritis and osteoarthritis indicate the need to implement public awareness campaigns aimed at reaffirming specialty against inflammatory joint disease.

Key words: perception, survey, rheumatology, rheumatoid arthritis.

\section{Correspondencia}

E-mail: Jorge.velasco@fundacionarticular.org 


\section{Introducción}

Las limitaciones funcionales sean por dolor, daño articular o por compromiso sistémico y/o afecciones intercurrentes son comunes en la evolución a largo plazo de la artritis reumatoidea. Con una morbimortalidad claramente aumentada ${ }^{1,2}$, el impacto personal, social y económico es considerable.

La lesión erosiva, signo característico de afectación articular, evolución y pronóstico, se encuentra presente en alrededor del $75 \%$ de los pacientes dentro de los primeros dos años de iniciada la enfermedad ${ }^{3}$.

Se encuentra bien establecido el hecho de que el abordaje diagnóstico y terapéutico tempranos beneficia significativamente la evolución de la AR tanto en términos clínicos como radiológicos ${ }^{4-12}$. Así, el inicio oportuno del tratamiento con fármacos antirreumáticos modificadores de la enfermedad (FAME) dentro de los 3 meses de comenzada la enfermedad fue señalado como el mejor predictor de remisión ${ }^{13,14}$, estableciéndose el concepto de ventana de oportunidad terapéutica. Una demora de solo 8 o 9 meses tendría implicancias en términos de evolución clínica ${ }^{15}$. De tal manera, que el inicio tardío del tratamiento en sí mismo constituye un factor de mal pronóstico.

Considerando la relevancia clínica del diagnóstico temprano emerge, aunque aún sin definiciones universalmente aceptadas, el concepto de AR temprana y muy temprana (ERA y VERA, sus acrónimos en inglés), esto es, menos de 3 meses o 6 semanas respectivamente del inicio de los síntomas; la intervención terapéutica dentro de estos períodos impacta fuertemente en el pronóstico de la afección ${ }^{16}$.

La importancia del diagnóstico y tratamiento tempranos fue enfatizada por la Liga Europea contra el Reumatismo (EULAR) quien recomienda que los pacientes con artritis de más de una articulación deberían ser derivados al reumatólogo, idealmente, dentro de las 6 semanas del comienzo de los síntomas. La clasificación que proponen incorpora la posibilidad del diagnóstico temprano de la enfermedad ${ }^{17,18}$. Asimismo, se señalan parámetros de alertas tempranas, tales como número de articulaciones inflamadas o duración de la rigidez matinal ${ }^{19}$.

Por práctica clínica y estudios formales, el mundo reumatológico comprende, desde hace tiempo, la importancia del diagnóstico y tratamientos tempranos ${ }^{20}$. La incorporación precoz y adecuada de FAME y una mejor evolución clínica, fueron observadas cuando tal actitud terapéutica estuvo en manos de reumatólogos ${ }^{21-24}$.

A pesar de lo expuesto, se ha señalado que la mitad de los pacientes no son derivados dentro de los 6 meses de iniciada la enfermedad y, probablemente, no estén recibiendo tratamiento adecuado ${ }^{25-27}$. Asimismo, el porcentaje de pacientes asistido por reumatólogos dentro de las 12 semanas del comienzo de la afección varía desde el
$1 \%$ hasta el $50 \%$ según el país o institución de referencia ${ }^{13,26}$. La demora promedio en la asistencia especializada desde el inicio de los síntomas fue estimada en 6 meses en 10 países europeos, con un porcentaje de diagnóstico menor a 3 meses de entre el $8 \%$ y el $42 \%{ }^{27}$. Podríamos afirmar, entonces, que la demora en la asistencia reumatológica es la constante más que la excepción ${ }^{25,28,29}$.

La observancia de tales dilaciones condujo a adoptar diversas estrategias conducentes al diagnóstico temprano apuntando a AR y/o artritis indiferenciada ${ }^{32}$. Se reportaron diferentes iniciativas tales como campañas de detección en ferias de salud ${ }^{39}$, cuestionarios autoadministrados en vía pública, en atención primaria de salud ${ }^{33} \mathrm{y}$ en formato electrónico (Internet, redes sociales) ${ }^{34}$, workshops, teleclínicas, capacitación de médicos generalistas ${ }^{35}$, servicios de pronta atención en el marco de la atención primaria ${ }^{36}$, etc. La organización de clínicas de artritis temprana se encuentra en esa línea de asistencia reumatológica con demoras mínimas ${ }^{37-40}$.

Explicar aquellas demoras es complejo y no pueden atribuirse a una razón única. Se identificaron factores relacionados a pacientes, médicos generalistas, sistemas de salud y aún a los propios reumatólogos ${ }^{30,31}$.

La demora vinculada con el paciente en solicitar asistencia médica fue señalada como una de las más significativas en la postergación en el inicio del tratamiento ${ }^{41}$. Se estimó que en el Reino Unido, entre la mitad y tres cuartos de los pacientes con AR demorarán el contacto con su médico generalista en los primeros tres meses después de la iniciación de síntomas, y el 20\% demora más de un año ${ }^{41,42}$.

Las razones de tales comportamientos son multifactoriales, algunos vinculados a las características de la afección: pacientes con síntomas articulares asimétricos y de inicio brusco tienden a buscar ayuda médica antes que aquellas con un inicio simétrico y gradual ${ }^{43,44}$. Otras se vinculan a la creencia de que los síntomas son transitorios o se asocian a factores estresantes, a esfuerzos físicos, al clima o a la alimentación; confusión con artrosis; búsqueda de consejos de familiares o amigos; la automedicación; que "nada puede hacerse" ${ }^{\text {* }} \mathrm{o}$, simplemente, al desconocimiento de la enfermedad ${ }^{45,50}$.

A pesar de la frecuencia de las afecciones del aparato locomotor, no parecería haber temor por padecerlas ${ }^{50}$. Por tanto, no es posible concluir ciertamente qué actitud tomaría quien responde si realmente padeciera síntomas de AR. Sin embargo, considerando que la demora del paciente artrítico en la consulta con el especialista se ve influenciada por la opinión de familiares y amigos ${ }^{46}$, el conocimiento general de la afección surge como fuertemente influyente.

Considerando esta última afirmación, se diseñó el presente estudio con el objeto de explorar la percepción en la población general acerca del reumatólogo como opción frente a los síntomas de la enfermedad. 


\section{Material y métodos}

Diseño del estudio: observacional, analítico, transversal, muestreo aleatorio de individuos en la vía pública, encuesta de preguntas de respuestas mixtas (abiertas y cerradas). La petición de colaboración fue explícita y se suministró información de quién y por qué realiza la encuesta, se prestó especial interés en cuidar el anonimato de los encuestados. Estos debían ser personas mayores de 18 años sin vínculos con el encuestador.

El estudio de campo poseyó las siguientes características:

Ámbito: ciudad de Quilmes y Berazategui, sur del Gran Buenos Aires

Período de tiempo: 14 meses

Universo: ambos sexos, mayores de 18 años

Tamaño de la muestra: 1073 sujetos

Puntos de muestreo: vía pública

Fecha de realización de la encuesta: junio 2013 - julio 2014

Las encuestas fueron diseñadas con el objeto de relevar diferentes niveles de datos e información:

Datos demográficos (edad, sexo)

A) Si usted tuviera dolor e inflamación de los nudillos y muñecas de ambas manos: ¿a qué profesional consultaría?

B) ¿Usted padece estos síntomas?

C) ¿Conoce la diferencia entre artritis y artrosis?

Estas fueron realizadas por seis voluntarias entrenadas a través de capacitación teórico prácticas y juego de roles. Se realizó supervisión periódica por reumatólogos.

\section{Resultados}

Después de descartar aquellas planillas de encuestas con datos insuficientes (formulario incompleto), ambiguos (ej., dos casilleros tildados) o inadecuados (ej., edad menor de 18 años), se evaluaron las respuestas de 1046 personas (694 mujeres). (Gráfico 1)

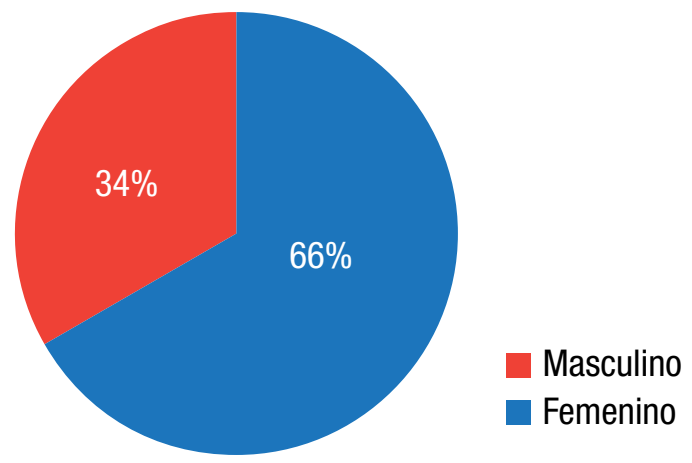

Gráfico 1. Encuestados según sexo
Se establecieron los siguientes rangos etarios: 18-30, 31-50, 51-80 y mayor de 81 años. La distribución de los grupos se muestra en la Tabla 1.

\begin{tabular}{|l|c|c|}
\hline Edad & (n) & Porcentaje \\
\hline $18-30$ & 268 & 25,62 \\
\hline $31-50$ & 308 & 29,45 \\
\hline $51-80$ & 441 & 42,16 \\
\hline mayor de 81 & 29 & 2,77 \\
\hline Total & 1046 & 100,00 \\
\hline
\end{tabular}

Tabla 1.

\section{Resultados generales}

A. Si Ud tuviera dolor e inflamación en los nudillos y muñecas de ambas manos ¿a qué profesional consultaría?

El médico traumatólogo fue la opción más seleccionada: $61 \%(635 / 1046)$ de los casos, el clínico en el 17\% (181/1046), el reumatólogo en el 15\% (157/1046), otros $7 \%$ (74/1046). (Gráfico 2)

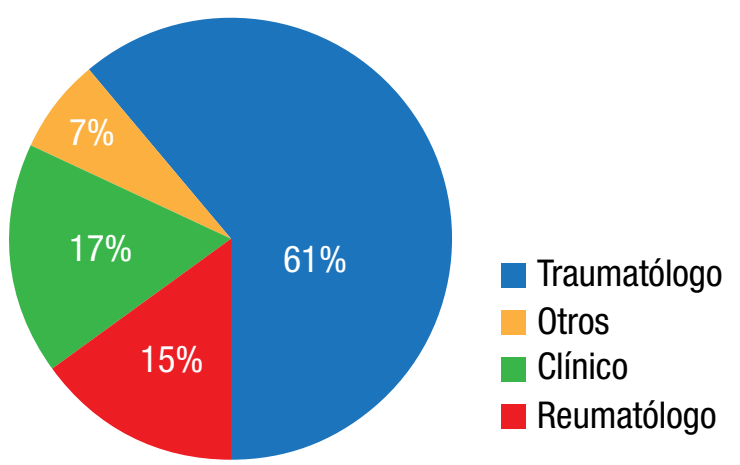

\section{Gráfico 2. Elección del especialista}

\section{B. ¿Usted padece estos sintomas?}

Las respuestas incluían dos opciones (SÍ/NO). Optaron por la afirmativa el $33 \%(344 / 1046)$ y el $67 \%$ por la negativa (702/1046). (Gráfico 3)

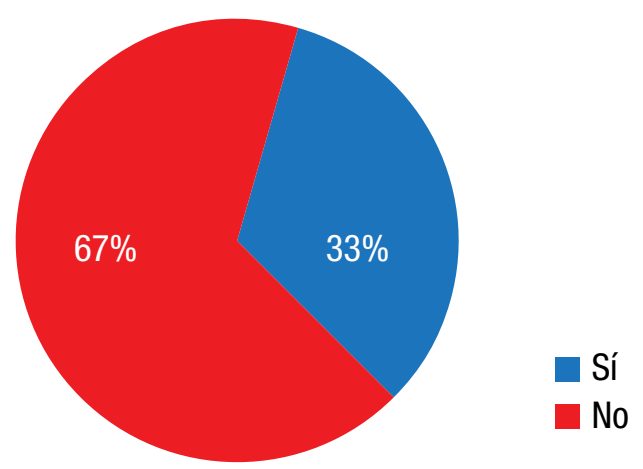

Gráfico 3. ¿Ud. padece estos síntomas? 
C. ¿Conoce la diferencia entre artritis y artrosis?

Las respuestas podían ser por SÍ o por NO. Por la afirmativa optó el $19 \%(205 / 1046)$ y por la negativa el $81 \%$ (841/1046). (Gráfico 4)

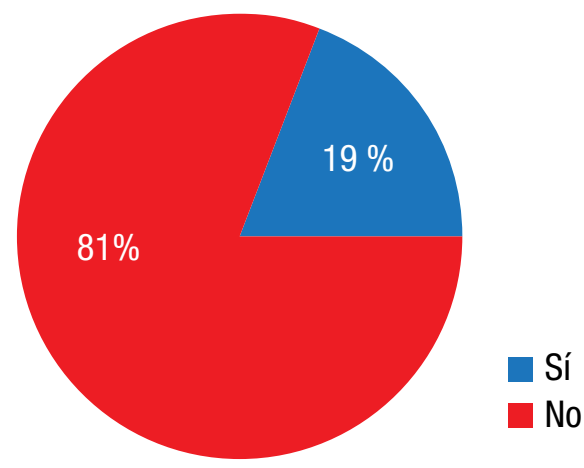

Gráfico 4. ¿Sabe la diferencia entre AR y artrosis?

\section{Segmentación por sexos}

\section{Femeninos}

Se observaron los siguientes resultados a partir de 694 femeninos:

A) Si usted tuviera dolor e inflamación en los nudillos y munecas de ambas manos: ¿a quién consultaría?

Traumatólogo $58 \%$, clínico $17 \%$, reumatólogo $18 \%$, otros $7 \%$. (Gráfico 5 )

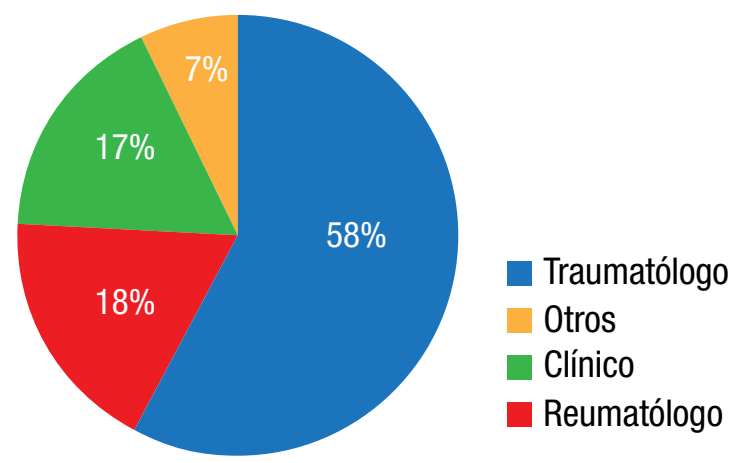

Gráfico 5. ¿A quién consultaría (femeninos)?

B) ¿Usted padece estos sintomas?

Sí: $36 \%$

No: $64 \%$

C) ¿Conoce la diferencia entre artritis y artrosis?

Sí: $21 \%$

No: $79 \%$

\section{Masculinos}

Respecto de la población masculina (352) se obtuvo la siguiente información:
A) Si usted tuviera dolor e inflamación en los nudillos y muñecas de ambas manos: ¿a quién consultaría?

Traumatólogo $66 \%$, clínico $18 \%$, reumatólogo 10\%, otros $6 \%$. (Gráfico 6 )

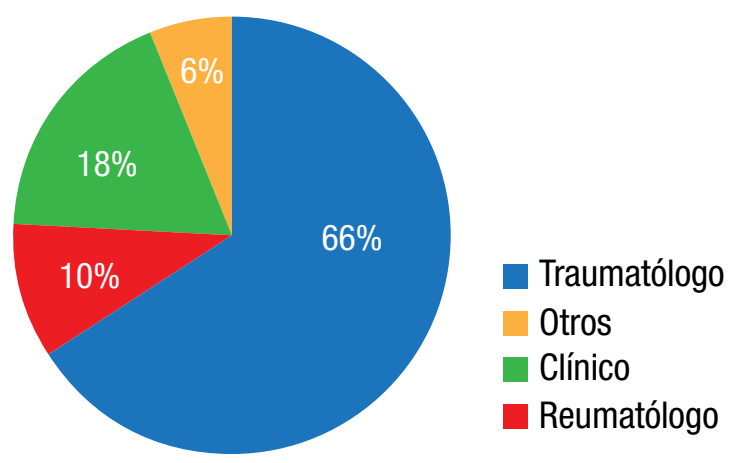

Gráfico 6. ¿A quién consultaría (masculinos)?

$B)$ ¿Usted padece estos sintomas?

Sí: $26 \%$

No: $74 \%$

C) ¿Conoce la diferencia entre artritis y artrosis?

Sí: $17 \%$

No: $83 \%$

El resumen de los datos obtenidos de la segmentación por sexos se muestra en la Tabla 2 y en el Gráfico 7.

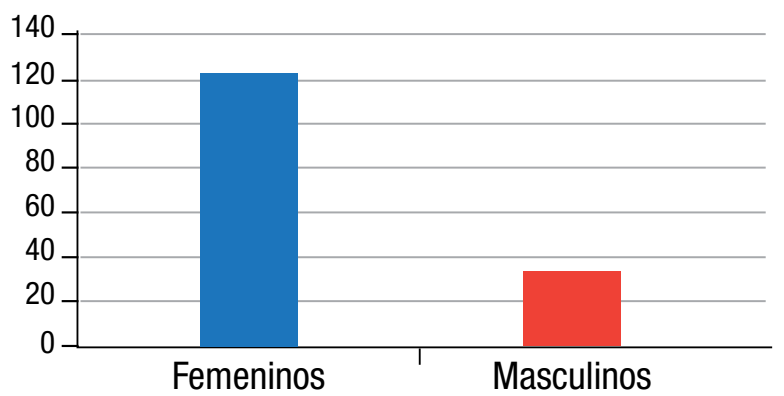

Gráfico 7. Número de encuestados por población femenina y masculina que consultaría a un reumatólogo.

\begin{tabular}{|l|l|l|l|l|l|l|l|l|}
\cline { 2 - 10 } \multicolumn{1}{c|}{} & \multicolumn{4}{|l|}{ A (\%) } & \multicolumn{2}{c|}{ B (\%) } & \multicolumn{2}{c|}{ C (\%) } \\
\cline { 2 - 10 } \multicolumn{1}{c|}{} & T & C & R & 0 & Sí & No & Sí & No \\
\hline Femeninos & 58 & 17 & $\mathbf{1 8}$ & 7 & 36 & 64 & 21 & 79 \\
\hline Masculinos & 66 & 18 & $\mathbf{1 0}$ & 6 & 26 & 74 & 17 & 83 \\
\hline
\end{tabular}

Tabla 2. $A, B, C$ refieren a las preguntas realizadas. T: traumatólogo, C: clínico: $R$ : reumatólogo, 0: otros.

\section{Segmentación por edades}

Al segmentar por edades el universo de encuestados, el grupo etario que más elegiría al reumatólogo fue el de mayor a 81 años. Este mismo grupo fue el que más refería 
poseer síntomas. (Tabla 3)

\begin{tabular}{|l|l|l|l|l|l|l|l|l|}
\cline { 2 - 9 } \multicolumn{1}{c|}{} & \multicolumn{4}{|c|}{ A (\%) } & \multicolumn{2}{c|}{ B (\%) } & \multicolumn{2}{c|}{ C (\%) } \\
\cline { 2 - 9 } \multicolumn{1}{c|}{} & T & C & R & 0 & Sí & No & Sí & No \\
\hline $18-30$ & 66 & 18 & $\mathbf{1 0}$ & 7 & 26 & 74 & 17 & 83 \\
\hline $31-50$ & 59 & 23 & $\mathbf{1 3}$ & 5 & 31 & 69 & 15 & 85 \\
\hline $51-80$ & 58 & 16 & $\mathbf{2 0}$ & 6 & 46 & 54 & 28 & 54 \\
\hline Mayor de 81 & 52 & 21 & $\mathbf{2 4}$ & 3 & 62 & 38 & 17 & 83 \\
\hline
\end{tabular}

Tabla 3. $A, B$ y $C$ refieren a las preguntas del cuestionario. $T$ : traumatólogo, C: clínico, $R$ : reumatólogo, 0: otros.

\section{Segmentación por percepción de síntomas}

Se analizaron los datos obtenidos de acuerdo a la percepción o no de síntomas vinculados. De los que refirieron padecer síntomas (352 personas), el 25\% consultaría al reumatólogo en oposición al 13\% de los que no los referían (695 personas). (Tabla 4, Gráficos 8 y 9)

\begin{tabular}{|l|l|l|l|l|l|l|}
\cline { 2 - 7 } \multicolumn{1}{c|}{} & \multicolumn{3}{c|}{ A (\%) } & \multicolumn{2}{c|}{ B (\%) } \\
\cline { 2 - 7 } \multicolumn{1}{c|}{} & T & C & R & 0 & Sí & No \\
\hline Con síntomas & 55 & 16 & $\mathbf{2 5}$ & 4 & 28 & 72 \\
\hline Sin síntomas & 59 & 23 & $\mathbf{1 3}$ & 5 & 31 & 69 \\
\hline
\end{tabular}

Tabla 4. $A$ y $B$ refieren a las preguntas del cuestionario. $T$ : traumatólogo, C: clínico, $R$ : reumatólogo, 0: otros.

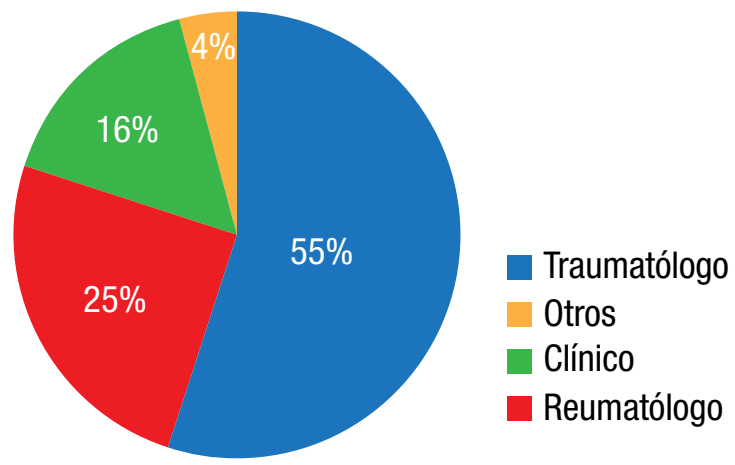

Gráfico 8. Refiere síntomas.

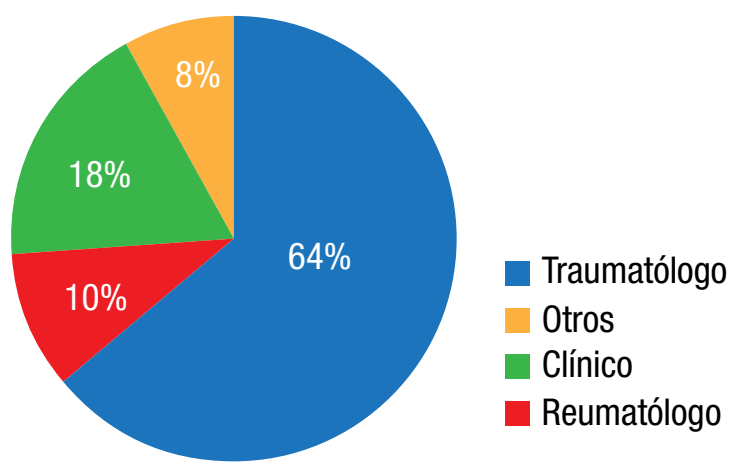

Gráfico 9. No refiere síntomas.
V. Segmentación por conocimiento en la diferencia entre artritis y artrosis

De los 205 encuestados que referían conocer la diferencia entre artritis y artrosis, el $42 \%$ elegiría al reumatólogo en caso de padecer síntomas sugestivos de AR. Contrariamente, de los 841 sujetos que refería no conocerla, el $9 \%$ hubiera elegido a un especialista en reumatología. (Tabla 5, Gráficos 10 y 11)

\begin{tabular}{|l|l|l|l|l|}
\cline { 2 - 5 } \multicolumn{1}{c|}{} & \multicolumn{4}{c|}{ A (\%) } \\
\cline { 2 - 5 } \multicolumn{1}{c|}{} & T & C & R & 0 \\
\hline Refiere conocer & 43 & 10 & $\mathbf{4 2}$ & 5 \\
\hline Refiere desconocer & 66 & 18 & $\mathbf{9}$ & 7 \\
\hline
\end{tabular}

Tabla 5 . Referencias. T: traumatólogo, C: clínico, R: reumatólogo, $0:$ otros. A: refiere a la pregunta del cuestionario.

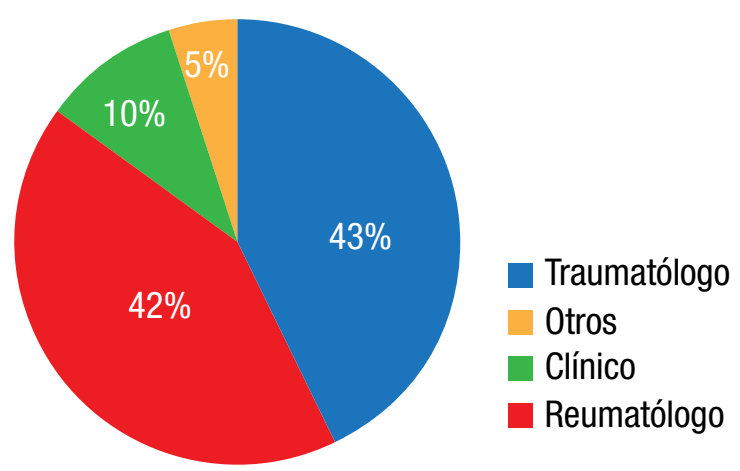

Gráfico 10. Refiere conocer diferencia entre $A R$ y $O A$

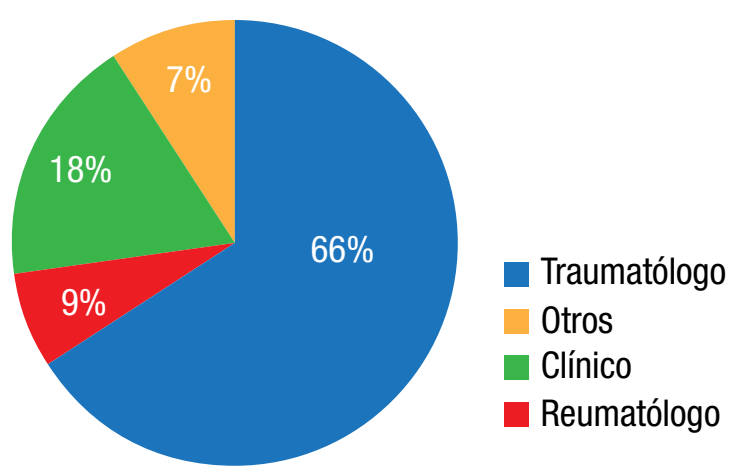

Gráfico 11. Refiere desconocer diferencia entre $A R$ y $O A$

\section{Discusión}

El diagnóstico y tratamientos tempranos de la AR constituyen un objetivo sanitario ineludible. Sin embargo, colisiona con las demoras observadas en diferentes países y ámbitos de la salud identificándose obstáculos significativos en todos los niveles intervinientes (pacientes, profesionales, efectores de salud) en el acceso temprano al 
reumatólogo.

La desinformación, el desconocimiento y la confusión son factores que contribuyen fuertemente a la demora en el diagnóstico de la AR. Una encuesta británica mostró que casi la mitad de los entrevistados referían conocer la $\mathrm{AR}$, pero al preguntar más profundamente solo el $5 \%$ la identificaron como una enfermedad inflamatoria. ${ }^{48}$ Otra evaluación realizada por el gobierno británico mostró cifras sugestivas: dos de cada tres personas entrevistadas creían que la AR era causada por desgaste articular ${ }^{49}$.

Tal confusión no parecería ser privativa de la comunidad general. Speed C. y cols. evaluaron formularios de derivación de los médicos generalistas a los servicios de Traumatología y Reumatología del Hospital de Addenbrooke en Cambridge, Inglaterra. Sobre 1087 derivaciones hallaron que el $27 \%$ de las interconsultas con el traumatólogo referían a patologías que definitiva o probablemente deberían ser vistas por un reumatólogo. Inversamente, el $2 \%$ de las reumatológicas debían haber sido derivadas a un traumatólogo ${ }^{46}$.

El Departamento de Comunicación de la Universidad de Twente, Holanda, encuestó a 658 personas (ninguna de ellas con afecciones articulares) respecto de la actitud y conocimiento de las enfermedades reumáticas. La mitad de los encuestados respondieron incorrectamente a preguntas dirigidas a la percepción general de estas afecciones, aunque mostraron deseos de mayor información; 4 de 10 desconocía que la AR es una enfermedad articular inflamatoria; el $70 \%$ creía que la AR era producida por una dieta pobre o factores ambientales ${ }^{50}$.

Asimismo, el desconocimiento de la AR como afección relevante también se evidenció en la encuesta de la National Rheumatoid Arthritis Society (Reino Unido), la que mostró que solo el $10 \%$ de la población estaba convencida de conocer los síntomas de la AR. El 60\% consideró que no consultaría a un profesional en caso de padecer alguna afección reumática, incluso aquellos con experiencia directa con la enfermedad mostraron un llamativo nivel de desconocimiento de la misma ${ }^{51,52}$.

Nuestra encuesta se enfocó en un escenario ex ante a fin de explorar la percepción de la población en un escenario potencial de sintomatología compatible con AR. La pregunta dirigida a ello era de respuesta abierta, por lo que la elección del especialista no estaba previamente condicionada. La proporción de individuos que optó por el reumatólogo fue sorprendentemente baja (15\%), aunque muy similar a la elección del clínico (17\%); el traumatólogo fue el especialista más elegido $(61 \%)$ por la población encuestada.

En nuestro estudio, la elección del reumatólogo como opción frente a los síntomas compatibles con AR aumentó a medida en que se consideraban grupos etarios mayores. Este hallazgo fue inverso a la elección del traumatólogo. Aunque con un número pequeño de encuestados (29), el grupo mayor de 81 años fue en el que se encontró mayor participación relativa en la elección del reumatólogo (24\%), aún así no alcanzó a superar a la del traumatólogo $(52 \%)$. Asimismo, las mujeres y quienes referían percibir síntomas también fueron grupos que mostraron mayor tendencia a la consulta con el reumatólogo. De la combinación de las respuestas de los diferentes subgrupos surge que quienes más elegirían al reumatólogo $(56 \%)$ fueron del sexo femenino de entre 51 y 80 años y que conocían la diferencia entre AR y OA.

Es sabido que la AR afecta aproximadamente al $1 \%$ de la población; sorpresivamente la afirmativa a la pregunta “¿usted padece estos síntomas?" fue del $33 \%$. Naturalmente, no podríamos inferir que tal frecuencia responda a la presencia de síntomas de AR, más bien lo consideramos como un hallazgo indirecto del desconocimiento de la enfermedad. Cierta similitud mostró una encuesta reciente de la británica National Rheumatoid Arthritis Society en la que se mostró que el $36 \%$ creía que ellos o sus allegados más cercanos se encontraban afectados directamente por $\mathrm{AR}^{52}$. Una evaluación previa mostró que más de la mitad de los encuestados acordaba padecer la enfermedad aunque solo el 10\% confiaban en conocer los síntomas de la afección ${ }^{51}$.

En nuestra población, aproximadamente 4 de cada cinco encuestados desconocía la diferencia entre artritis y artrosis, hallazgo muy similar a lo observado en una encuesta contemporánea ${ }^{52}$. Apreciamos desigualdades entre los diferentes grupos etarios; el que más refería conocer aquella diferencia fue el de 51-80 años (28\%), aunque curiosamente casi la mitad de los respondedores refería padecer la enfermedad. Coincidente con lo observado en la bibliografía consultada, la población más joven fue quien menos refería distinguir entre artritis y artrosis. El hallazgo sería consistente con un estudio reciente en el que se comprobó que los pacientes con patología reumática de entre 20 y 29 años fueron los que más demoraron la consulta al especialista ${ }^{55}$. Estos datos son significativos al momento de seleccionar los medios de concientización pública, debiéndose considerar las formas modernas de comunicación.

El grupo que refería conocer la diferencia entre artritis y artrosis fue quien más consultaría al reumatólogo (42\% vs $9 \%$ ). Indirectamente, se podría inferir, aunque no categóricamente, que el conocimiento básico debería impactar en una mayor concientización pública.

La percepción popular del "reuma" como una afección de personas mayores conduciría a subestimar la posibilidad de considerar otras enfermedades del aparato locomotor así como otros grupos etarios afectados. Van Der Wardt y cols. encontraron que casi el $80 \%$ de los encuestados desconocía que existían más de 100 enfermedades reumáticas ${ }^{50}$.

En la búsqueda de una interpretación convincente, deberíamos intentar interpretar un fenómeno a priori de aquello. Literalmente, la reumatología es el estudio (logos) del fluir de los humores (del griego rheó, rhuó: correr, fluir, destilar) ${ }^{54}$. Tal denominación no parecería ser la más adecuada siendo intrínsecamente confusa a la luz del conocimiento actual de la especialidad y de sus alcances. Aún el diccionario de la Real Academia Española considera a los "reumatismos" desde una óptica limitada: "Enfermedad que se manifiesta generalmente por inflamación de las articulaciones de las extremidades" 


\section{Conclusiones}

El abordaje temprano de la AR fue señalado como un sólido factor de predicción de su evolución, estableciéndose el concepto de ventana de oportunidad terapéutica. Asimismo, el diagnóstico, tratamiento y seguimiento en manos de especialistas en reumatología mostró correlación con un mejor pronóstico.

A pesar de ello, el retraso en la asistencia reumatológica se convirtió en una constante que, pareciera, posee alcances globales. Se señalaron diversas razones para explicar este fenómeno, indicándose desde factores relacionados con el paciente hasta los propios del sistema de salud.

Nuestra encuesta indagó acerca de la percepción que posee la población fuera del contexto asistencial en un escenario potencial de sintomatología compatible con AR. El reumatólogo fue elegido por la minoría de los encuestados, siendo el traumatólogo el especialista que más fue señalado en el supuesto escenario de inflamación articular. Asimismo, coincidente con la bibliografía consultada, se apreció un franco desconocimiento de la diferencia entre artritis y artrosis así como confusión respecto de la presencia de sintomatología compatible con la enfermedad.

Lo señalado reafirma la necesidad de continuar ejecutando campañas de concientización pública dirigidas, no solo a la identificación de pacientes con AR, sino a la reafirmación de la importancia de la especialidad frente a las patologías musculoesqueléticas.

\section{Agradecimientos}

A todas las voluntarias que participaron y a sus coordinadoras Sra. María Santoro y Lic. Lorena González.

\section{Declaración de conflictos de interés}

Los autores declaran no poseer conflictos de interés.

\section{Bibliografía}

1. Gabriel SE, Crowson CS, O'Fallon WM. Mortality in rheumatoid arthritis: have we made an impact in 4 decades? J Rheumatol 1999;26:2529-33.

2. Wolfe F, Mitchell DM, Sibley JT, Fries JF, Bloch DA, Williams CA, et al. The mortality of rheumatoid arthritis. Arthritis Rheum 1994;37:481-94.

3. van der Heijde DMFM. Radiographic imaging: the "gold standard" for assessment of disease progres- sion in rheumatoid arthritis. Rheumatology (Oxford) 2000;39(suppl 1):9-16.

4. Lard LR, Visser H, Speyer I, et al. Early versus delayed treatment in patients with recent-onset rheumatoid arthritis: comparison of two cohorts who received different treatment strategies. Am J Med. 2001; 111:446-51.

5. Mottonen T, Hannonen P, Korpela M, et al. Delay to institution of therapy and induction of remission using single-drug or combination-disease-modifying antirheumatic drug therapy in early rheumatoid arthritis. Arthritis Rheum. 2002; 46:894-8.

6. Nell VP, Machold KP, Eberl G, Stamm TA, Uffmann $\mathrm{M}$, Smolen JS. Benefit of very early referral and very early therapy with disease-modifying anti-rheumatic drugs in patients with early rheumatoid arthritis. Rheumatology (Oxford). 2004; 43:906-14.

7. Finckh A, Liang MH, van Herckenrode CM, de Pablo P. Long-term impact of early treatment on radiographic progression in rheumatoid arthritis: a meta-analysis. Arthritis Rheum. 2006; 55:864- 72.

8. Emery P, Kvien TK, Combe B, et al. Combination etanercept and methotrexate provides better disease control in very early ( $<=4$ months) versus early rheumatoid arthritis ( $>4$ months and $<2$ years): post hoc analyses from the COMET study. Ann Rheum Dis. 2012

9. Mottonen T, Hannonen P, Leirisalo-Repo M, et al. Comparison of combination therapy with single-drug therapy in early rheumatoid arthritis: a randomised trial. FIN-RACo trial group. Lancet. 1999; 353:1568-73.

10. Breedveld FC, Weisman MH, Kavanaugh AF, et al. The PREMIER study: a multicenter, randomized, doubleblind clinical trial of combination therapy with adalimumab plus methotrexate versus methotrexate alone or adalimumab alone in patients with early, aggressive rheumatoid arthritis who had not had previous methotrexate treatment. Arthritis Rheum. 2006; 54:26-37.

11. van der Linden MP, le Cessie S, Raza K, et al. Longterm impact of delay in assessment of patients with early arthritis. Arthritis Rheum. 2010; 62:3537-46.

12. Lard LR, Visser H, Speyer I, et al. Early versus delayed treatment in patients with recent-onset rheumatoid arthritis: comparison of two cohorts who received different treatment strategies. Am J Med 2001;111:446-51.

13. Elisa Gremese, Fausto Salaffi, Silvia Laura Bosello, Alessandro Ciapetti, Francesca Bobbio-Pallavicini, Roberto Caporali, Gianfranco Ferraccioli1 Very early rheumatoid arthritis as a predictor of remission: a multicentre real life prospective study Ann Rheum Dis 2013;72:858-862.

14. Cush JJ. Early rheumatoid arthritis - is there a window of opportunity? J Rheumatol Suppl. 2007; 80:1-7.

15. Emery, P. Evidence Supporting the Benefit of Early In- 
tervention in Rheumatoid Arthritis. J Rheumatol 2002; 29 Suppl 66: 3-8.

16. van der Helm-van Mil AH, le Cessie S, van Dongen $H$, Breedveld FC, Toes RE, Huizinga TW. A prediction rule for disease outcome in patients with recent-onset undifferentiated arthritis: how to guide individual treatment decisions. Arthritis Rheum. 2007; 56:433-40.

17. Combe B, Landewe R, Lukas $C$, et al. EULAR recommendations for the management of early arthritis: report of a task force of the European Standing Committee for International Clinical Studies Including Therapeutics (ESCISIT). Ann Rheum Dis 2007;66:3445.

18. Aletaha D, Neogi T, Silman AJ, et al. 2010 rheumatoid arthritis classifi cation criteria: an American College of Rheumatology/European League Against Rheumatism collaborative initiative. Ann Rheum Dis 2010;69:15808.

19. Emery P, Breedveld FC, Dougados M, et al. Early referral recommendation for newly diagnosed rheumatoid arthritis: evidence based development of a clinical guide. Ann Rheum Dis 2002;61:290-7.

20. Aletaha $D$, et al. Attitudes to early rheumatoid arthritis: changing patterns. Results of a survey Ann Rheum Dis 2004;63:1269-1275.

21. Criswell LA, Such CL, Yelin EH. Differences in the use of second-line agents and prednisone for treatment of rheumatoid arthritis by rheumatologists and nonrheumatologists. J Rheumatol 1997;24:2283-90.

22. Ward MM, Leigh JP, Fries JF. Progression of functional disability in patients with rheumatoid arthritis. Associations with rheumatology subspecialty care. Arch Intern Med 1993;153:2229-37.

23. Nanji J, Choi M, Ferrari, R, Lyddell C, Russell A, Time to consultation and disease-modifying antirheumatic drug treatment of patients with rheumatoid arthritis-northern Alberta perspective. J Rheumatol. 2012 Apr;39(4):707-11.

24. Widdifield J, Bernatsky S, Paterson JM, Thorne JC, Cividino A, Pope J, Gunraj N, Bombardier C. Quality care in seniors with new-onset rheumatoid arthritis: a Canadian perspective. Arthritis Care Res (Hoboken). $2011 \mathrm{Jan} ; 63(1): 53-7$.

25. van der Linden MPM, Le Cessie S, Raza K, et al. Long term impact of delay in assessments of patient with early arthritis. Arthritis Rheum 2010;62: 3537-46.

26. Irvine S, Munro R, Porter D. Early referral, diagnosis, and treatment of rheumatoid arthritis: evidence for changing medical practice. Ann Rheum Dis 1999;58:510-3.

27. Aletaha D, Eberl G, Nell VP, Machold KP, Smolen JS. Practical progress in realisation of early diagnosis and treatment of patients with suspected rheumatoid arthritis: results from two matched questionnaires within three years. Ann Rheum Dis 2002;61:630-4.

28. Raza K, Stack R, Kumar K, et al. Delays in assessment of patients with rheumatoid arthritis: variations across Europe. Ann Rheum Dis 2011;70:1822-5.

29. Hernández-García C, Vargas E, Abásolo L, et al. Lag time between onset of symptoms and access to rheumatology care and DMARD therapy in a cohort of patients with rheumatoid arthritis. J Rheumatol 2000;27:2323-8.

30. Woolf AD, Zeidler H, Haglund U, et al. Musculoskeletal pain in Europe: its impact and a comparison of population and medical perceptions of treatment in eight European countries. Ann Rheum Dis 2004;63:342-7.

31. Machold KP, Köller MD, Pfl ugbeil S, et al. The public neglect of rheumatic diseases: insights from analyses of attendees in a musculoskeletal disease awareness activity. Ann Rheum Dis 2007;66:697-9.

32. Villeneuve E, et al. A systematic literature review of strategies promoting early referral and reducing delays in the diagnosis and management of inflammatory arthritis Ann Rheum Dis 2013;72:13-22.

33. Bell MJ, Tavares R, Guillemin F, et al. Development of a self-administered early inflammatory arthritis detection tool. BMC Musculoskelet Disord 2010;11:50.

34. Hwang AS, Gall V, Liang MH. Evaluation of the internet for finding persons with undiagnosed rheumatoid arthritis and systemic lupus erythematosus. J Clin Rheumatol 2009;15:218-22.

35. Schulpen GJ, Vierhout WP, van der Heijde DM, et al. Joint consultation of general practitioner and rheumatologist: does it matter? Ann Rheum Dis 2003;62:15961.

36. Gärtner M, Fabrizii JP, Koban E, et al. Immediate access rheumatology clinic: effi ciency and outcomes. Ann Rheum Dis 2012;71:363-8.

37. van der Horst-Bruinsma IE, Speyer I, Visser H, et al. Diagnosis and course of earlyonset arthritis: results of a special early arthritis clinic compared to routine patient care. Br J Rheumatol 1998;37:1084-8.

38. Quinn MA, Emery P. Are early arthritis clinics necessary? Best Pract Res Clin Rheumatol 2005;19:1-17.

39. Deane KD, Striebich CC, Goldstein BL, et al. Identification of undiagnosed inflammatory arthritis in a community health fair screen. Arthritis Rheum 2009;61:1642-9.

40. Machold KP, Köller MD, Pfl ugbeil S, et al. The public neglect of rheumatic diseases: insights from analyses of attendees in a musculoskeletal disease awareness activity. Ann Rheum Dis 2007;66:697-9.

41. Kumar K, et al. Delay in Presentation to Primary Care Physicians is the Main Reason Why Patients With 
Rheumatoid Arthritis Are Seen Late by Rheumatologists. Rheumatology 2007;46(9):1438-1440.

42. Sandhu RS, et al. Comment On: Delay in Presentation to Primary Care Physicians is the Main Reason Why Patients with Rheumatoid Arthritis Are Seen Late by Rheumatologists. Rheumatology 2008;47:559-60.

43. Emery P. Evidence Supporting the Benefit of Early Intervention in Rheumatoid Arthritis. J Rheumatol 2002; 29 Suppl 66: 3-8.

44. Machold K. The public neglect of rheumatic diseases: insights from analyses of attendees in a musculoskeletal disease awareness Ann Rheum Dis 2007;66:697-9.

45. Stack, RJ, et al. Delays in Help Seeking at the Onset of the Symptoms of Rheumatoid Arthritis: A Systematic Synthesis of Qualitative Literature. Ann Rheum Dis 2012; 71: 493-497.

46. C. A. Speed and A. J. Crisp Referrals to hospital-based rheumatology and orthopaedic services: seeking direction Rheumatology 2005;44:469-471.

47. Steward K, Land M. The King found Perceptions of patients and professionals on rheumatoid arthritis care Jan 2009.

48. Low Public Awareness of Symptoms Of Arthritis, $2000 \mathrm{http}: / /$ www.ipsos-mori.com/

49. Medical News Today, 'Alarming Ignorance' About Rheumatoid Arthritis March 2009. http://www.medicalnewstoday.com/releases/142497.php

50. Elly M Van Der Wardt, Erik Taal, Johannes J Rasker.
The general public's knowledge and perceptions about rheumatic diseases. Ann Rheum Dis 2000;59:32-38.

51. National Rheumatoid Arthritis Society (NRAS). Ignorance of rheumatoid arthritis puts thousands at risk, Arthritis Today, 10 November 2011.

52. Breaking Down Barriers: Rheumatoid Arthritis and Public Awareness. Jun 2013. National Rheumatoid Arthritis Society www.nras.org.uk

53. http://lema.rae.es/drae/?val=reumatismos

54. Monlau Felipe Diccionario etimológico de la lengua castellana, 1856. pag 395 http://books.google. com.ar/books? id=BXgCAAAAQAAJ\&printsec $=$ frontcover\&dq=related:LCCN03024159\&redir $\mathrm{esc}=\mathrm{y} \# \mathrm{v}=$ onepage $\& \mathrm{q} \& \mathrm{f}=$ false

55. Milne V, Kearns R, Harrison A Patient age, ethnicity and waiting times determine the likelihood of non-attendance at a first specialist rheumatology assessment. J Rheum Dis 2014 Jan;17(1):19-25. doi: 10.1111/1756185X.12126. Epub 2013 Jun 20. 